\title{
Incidence of ventilator associated pneumonia
}

\section{Chandrakanth C.* Anushree and Vinod A.}

*Department of Microbiology, idar Institute of Medical Sciences \& Teaching Hospital, Bidar. 585401, Karnataka, India, drchandu_75@yahoo.com

Department of Pathology, Narayana Medical dranushree77@gmail.com, vinod.athuluri@gmail.com

College, Nellore,

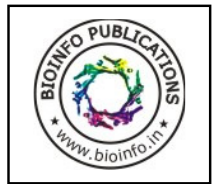

Abstract- Background: The development of VAP is also associated with greater hospital mortality rates and longer lengths of stay in Intensive Care Unit (ICU) and hospitals. A prospective study was conducted to find out the incidence of VAP and to identify the most prevalent pathogens causing VAP. The study was conducted during the period of September 2008 to March 2009 at ICU of Narayana Medical College and General Hospital. The endotracheal aspirates were collected andtransported to microbiology laboratory within 15 minutes, where they were processed and cultured as per the standard protocol and standard microscopic examinations were conducted. Statistical analysis was performed using SPSS 11.0 and Systat 8.0. Out of the 100 patients studied, 29 were found to have VAP. Among these patients, 32\% were reported to have hypertension, $29 \%$ were reported to have diabetes and $12 \%$ had both diabetes and hypertension. Gram negative organisms were predominant among the isolates accounting for $89 \%$. The rest were found to be gram positive organisms. Among gram negative organisms, Pseudomonas species, Klebsiella species and E.coli were responsible for highest number of VAP infections. The range of bacteria that cause VAP and their susceptibility patterns vary widely among hospitals and selection of initial antimicrobial therapy need to be tailored to each institution's local patterns of antimicrobial resistance.

Key words: VAP (Ventilator Associated Pneumonia), ICU, MV (Mechanical Ventilator)

\section{Background}

Incidence of Hospital acquired infection has drastically reduced with proper implementation of precautions in the hospital environment. However, several such (Nosocomial) infections are still encountered in various departments of a Hospital. Pneumonia results from microbial invasion of the normally sterile lower respiratory tract and lung parenchyma caused by a defect in host defenses, challenge by a particularly virulent microorganism, or an overwhelming inoculum. Nosocomial Pneumonia is the second most among them which is associated with the highest case /fatality ratio. Among different types of Nosocomial infections, ventilator associated pneumonia (VAP) continues to complicate the course of $8-28 \%$ of patients receiving mechanical ventilation (MV)[1].The mortality rate associated with VAP ranges from $24-50 \%$ and can escalate up to $76 \%$ based on specific settings and host-pathogen relationship[1]. The development of VAP is also associated with greater hospital mortality rates and longer lengths of stay in Intensive Care Unit (ICU) and hospitals. Microorganisms responsible for VAP may differ according to the population of patients in the ICU, the duration of hospital and ICU stays, and the specific diagnostic method(s) used [2, 3, 4]. The high rate of respiratory infections due to Gram negative bacilli in this setting has been repeatedly documented. Several studies have reported that more than $60 \%$ of VAP is caused by aerobic Gram negative bacilli. More recently, some investigators have reported that grampositive bacteria have become increasingly more common in this setting, with $S$. aureus being the predominant gram-positive isolate [5]. Although the incidence of VAP and the contribution of various micro-organisms to this have been studied extensively around the world, no such work has been carried out in south India. Aprospective study was conducted at Narayana Medical College and General Hospital to find out the incidence of VAP and to identify the most prevalent pathogens causing VAP.

\section{Methods}

The study was conducted during the period of September 2008 to March 2009 at ICU of Narayana Medical College and General Hospital. A total of 100 patients connected with mechanical ventilators for more than 48 hours were studied. A proforma was filled in detail for each patient with regardto his/her name, age, sex, Ip/no, ward/unit, Clinical diagnosis, indications on artificial ventilation, nature of sample collected for VAP, New symptoms after ventilator connected(after 48hrs), Empirical treatment given.

\section{Sample collection}

The endotracheal aspirates were collected by using a sterile 12 Gauge endotracheal suction catheter tube. Suction catheter is connected to the suction pump and passed through endotracheal tube. In case of purulent secretion the Endo Tracheal suction catheter tips were collected using sterile scissors into the sterile container (screw capped). In case of thick or dry secretions with clinical diagnosis of pneumonia, sterile normal saline was pushed into endotracheal tube. Then suction was done for the aspirate. The samples were transported to microbiology laboratory within 15 minutes. Specimens were processed and cultured as per the standard protocol as soon as they were obtained and standard microscopic examinations were conducted. 
Antimicrobial susceptibility was analyzed using Mueller-Hinton agar (hi-media) using, KirbyBauer disk diffusion method. The isolated colony was sub cultured in peptone water and compared with 0.5 Mac. Farlandsturbidity. Plates was inoculated with a sterile cotton swab dipped in the standardized suspension of organism. After inoculum has dried, specified antibiotic discs were placed $2 \mathrm{~cm}$ apart from each other with forceps and were incubated for 16-18 hours at 37 OC aerobically. The Zone size was interpreted according to the reference charts provided by manufacturer. The statistical software namely SPSS 11.0 and Systat 8.0 were used for the analysis of the data and Microsoft Word and Excel have been used to generate graphs, tables etc.

\section{Results}

Of the 100 patients studied, 29 were found to have VAP. Further among these patients, a higher percent of patients were reported to have early onset defined as development of VAP before 5 days of MV than late onset defined as development of VAP after 5 days of MV (Table 1).

Table 1-Comparison of Early and Late onset VAP

\begin{tabular}{|l|l|}
\hline Total number of cases studied. & 100 \\
\hline Number of VAP cases identified & 29 \\
\hline Early onset(<5days) cases & $16(55.17 \%)$ \\
\hline Late onset $(>5$ days) cases & $13(44.82 \%)$ \\
\hline
\end{tabular}

Association of diabetes and hypertension Among the patients studied, $32 \%$ of patients were reported to have hypertension, $29 \%$ were reported to have diabetes and $12 \%$ were reported to have both diabetes and hypertension. Further VAP was found to be higher among patients with hypertension (Fig.1).

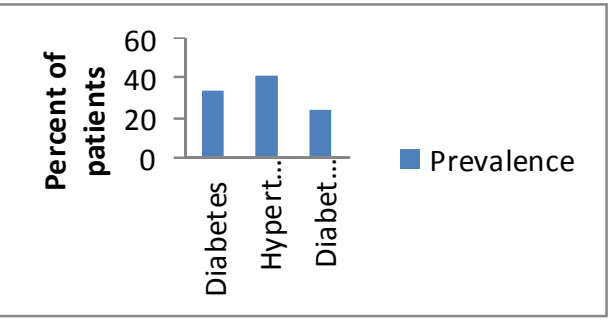

Fig. 1-Prevalence of diabetes and hypertension among patients

\section{Microorganisms isolated}

The causative organisms were isolated and identified. Gram negative organisms were predominant among the isolates accounting for $89 \%$. The rest were found to be gram positive organisms. Among gram negative organisms, Pseudomonas species, Klebsiella species and E.coli were responsible for highest number of VAP infections (Fig.2).

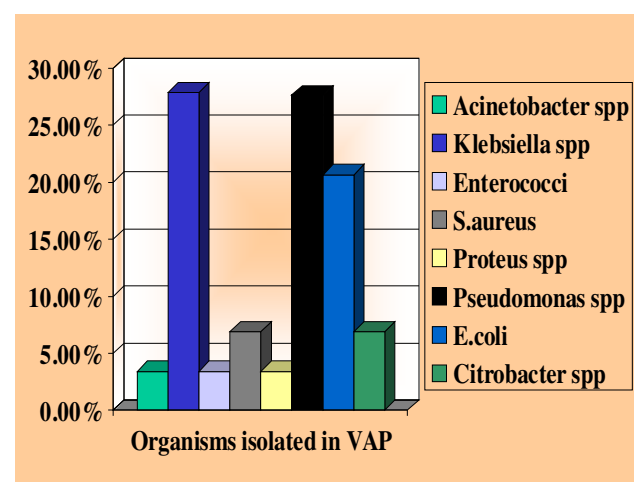

Fig. 2-Organisms isolated from patients with VAP

\section{Discussion}

Prolonged (more than 48 hours) MV is the most important factor associated with nosocomial pneumonia. However, VAP may occur within the first 48 hours after intubation. It is usual to distinguish hearly-onset VAP, which occurs during the first 5 days of MV, from late-onset VAP, which develops 5 or more days after initiationof MV. The incidence of the current study was $29 \%$ which was compared with $28 \%$ of incidence in a study by Fagon JY, et al. [1]. The onset of VAP in the current study was $55.17 \%$ in early onset which was comparable with $45 \%$ of early onset in a study by Prod'hom $G$ et.al. [6]. Not only are the causative pathogens commonly different but the disease is usually less severe and the prognosis better in early-onset than late-onset VAP. Tullu MS, et al. reported that E.coli, Klebsiella species and Pseudomonas species were isolated as the commonest organism colonizing the endotracheal tube leading to ventilator associated pneumonia. The current study also reported similar trend in isolates responsible for VAP. The frequency of isolation of Pseudomonas species (27.58\%), Acinetobacter $(3.44 \%)$, Klebsiella species $(27.58 \%)$, E.coli (20.68\%), Citrobacter (6.89\%), Proteus species $(3.44 \%)$, S.aureus $(6.89 \%)$ and Enterococci (3.44\%) were found to be comparable with that of study done by Chastre $\mathrm{J}$ etal[1] where the frequency of isolates of Pseudomonasspecies (24.4\%), Acinetobacter (7.9\%), Klebsiella species (15.6\%), E.coli (24.1\%) and Citrobacter (5\%). National nosocomial infections surveillance (NNIS) report stated that Pseudomonas aeruginosa have become class I cephalosporinase producers and are resistant to piperacillin and ceftazidime. Klebsiella species and other Enterobacteriaceae strains are also increasingly being recognized as producers of transferable extended spectrum beta-lactamases, which confer resistance to third generation cephalosporins. However, in current study Pseudomonasspecies is sensitive to cefoperazone +sulbactum, piperacillin, carbenicillin and imipenem. And most of the Enterobacteriaceae members are sensitive to amikacin, ciprofloxacin, cefeperazone + 
sulbactum. However, because the range of bacteria that cause VAP and their susceptibility patterns vary widely among hospitals in the same or different countries, selection of initial antimicrobial therapy need to be tailored to each institution's local patterns of antimicrobial resistance.

\section{References}

[1] Chastre J. and FagonJ.Y. (2002) Am. J. Respir. Crit. Care Med., 165 (7), 867-903.

[2] Fagon J.Y., Chastre J., Domart Y., Trouillet J.L., Pierre J., Darne C., et al. (1989) Am Rev Respir Dis., 139: 877 884.

[3] Torres A., Aznar R., Gatell J.M., Jimenez P., Gonzalez J., Ferrer A., et al. (1990) Am Rev Respir Dis., 142: 523-528.

[4] Rello J., Ausina V., Ricart M., Castella J., Prats G. (1993) Chest., 104:12301235.

[5] Spencer R.C. (1996) Eur J ClinMicrobiol Infect Dis., 15:281-285.

[6] Prod'hom G., Leuenberger P., Koerfer J., Blum A., Chiolero R., Schaller M.D., et al. (1994) Ann Intern Med., 120:653-662. 